Proc. Indian Acad. Sci. (Math. Sci.) Vol. 117, No. 2, May 2003, pp. 177-183.

Printed in India

\title{
Ergodic theory of amenable semigroup actions
}

\author{
ALI GHAFFARI \\ Department of Mathematics, Semnan University, Semnan, Iran
}

E-mail: ghaffari1380@yahoo.com

MS received 7 September 2005; revised 23 January 2007

Abstract. In this paper, among other things, we state and prove the mean ergodic theorem for amenable semigroup algebras.

Keywords. Asymptotically invariance property; Banach algebras; locally compact semigroup; mean ergodic theorem; topologically left invariant mean; topologically right invariant mean; weakly almost periodic.

\section{Introduction}

Actions of amenable semigroups in connection with fixed point conditions were considered in [8]. The classical mean ergodic and pointwise erogdic theorems are concerned with the convergence of the sequence of Cesaro means [14]. The mean ergodic theorem for amenable locally compact groups was proved by Greenleaf [9]10] (for more on mean ergodic theorem on locally compact group, the reader is referred to [10] and [14]). For the pointwise ergodic theorem, refer [14].

Our primary concern in this paper is to prove a theorem for an amenable locally compact semigroup $S$. Other aspects of the relationship between amenability and ergodic theory are mentioned in Corollaries 1, 2. A detailed historical account on the notion of ergodicity is given in [13].

Let $S$ be a locally compact Hausdorff semitopological semigroup. Let $M(S)$ be the Banach algebra of all bounded, regular Borel measures on $S$ with total variation norm and convolution as multiplication, and let $M_{0}(S)$ be the semigroup of all probability measures in $M(S)$. Let $M(S)^{*}$ be the continuous dual of $M(S)$, and 1 the linear functional in $M(S)^{*}$ such that $\langle 1, \mu\rangle=\mu(S)$ for all $\mu$ in $M(S)$.

Recall that on $M(S)^{* *}$ we define the first Arens product by

$$
\langle f \mu, v\rangle=\langle f, \mu * v\rangle, \quad\langle F f, \mu\rangle=\langle F, f \mu\rangle, \quad\langle G F, f\rangle=\langle G, F f\rangle
$$

where $\mu, v \in M(S), f \in M(S)^{*}$ and $F, G \in M(S)^{* *}$. A linear functional $M \in M(S)^{* *}$ is called a mean if $\langle M, f\rangle \geq 0$ whenever $f \geq 0$ and if $\langle M, 1\rangle=1$. A mean $M$ is topological left (right) invariant if $\langle M, f \mu\rangle=\langle M, f\rangle(\langle M, \mu f\rangle=\langle M, f\rangle)$ for any $\mu \in M_{0}(S)$ and $f \in$ $M(S)^{*}$. We know that topologically left invariant means on $M(S)^{*}$ have been studied by Riazi and Wong [16] and by Wong [18[19]20]. We shall follow [8] and [16] for definitions and terminologies not explained here. 


\section{Main results}

Throughout the paper, $S$ is a locally compact Hausdorff semitopological semigroup. A semigroup $S$ is said to act on a set $X$ (from the right) if there exists a mapping

$$
(\xi, s) \mapsto \xi s, X \times S \rightarrow X
$$

such that $\xi(s t)=(\xi s) t$ whenever $s, t \in S$ and $\xi \in X ; S$ is a transformation semigroup on $X$. We say that the complex Banach space $X$ with a mapping $(\xi, s) \mapsto \xi_{s}$ constitutes a right Banach $S$-module if the mapping has the following properties:

(a) for each fixed $s \in S$, the mapping $\xi \mapsto \xi s$ is linear on $X$;

(b) there exists a positive constant $k$ such that $\left\|\xi_{s}\right\| \leq k\|\xi\|(\xi \in X, s \in S)$ and, for every $\xi \in X$, the mapping $s \mapsto \xi s$ is continuous.

We define similarly a left dual $S$-module structure on $X^{*}$ by putting $\langle s f, \xi\rangle=\langle f, \xi s\rangle$ whenever $s \in S, \xi \in X$ and $f \in X^{*}$. For every $f \in X^{*}, s \mapsto s f$ is a continuous mapping of $S$ into $X^{*}$ when $X^{*}$ is equipped with the weak*-topology.

Let $X$ be a right Banach $S$-module. We may give $X$ a right Banach $M(S)$-module structure via a vector-valued integral. We put

$$
\xi \mu=\int \xi s \mathrm{~d} \mu(s)
$$

for $\mu \in M(S), \xi \in X$. Notice also that if $s \in S$ and $\xi \in X$, then $\xi \delta_{s}=\xi s$. Finally, for every $\mu \in M(S), \xi \in X$ and $f \in X^{*}$, we define $\langle\mu, f \xi\rangle=\langle f, \xi \mu\rangle$.

\section{PROPOSITION 1.}

Let $\xi \in X$ be such that $C_{\xi}=\overline{c o}\left\{\xi \delta_{s} ; s \in S\right\}$ is weakly compact. Then $C_{\xi}=\overline{\left\{\xi \mu ; \mu \in M_{0}(S)\right\}}$.

Proof. It is clear that $C_{\xi} \subseteq \overline{\left\{\xi \mu ; \mu \in M_{0}(S)\right\}}$. We will see that this inclusion is actually an equality. To reach a contradiction, assume that some $\xi \mu \in\left\{\xi \mu ; \mu \in M_{0}(S)\right\}$ is not in $C_{\xi}$. Theorem 3.4(b) of [17] shows that there exist $f \in X^{*}$ and $\gamma \in \mathbb{R}$ such that, for every $s \in S$,

$$
\operatorname{Re}\left\langle f, \xi_{s}\right\rangle<\gamma<\operatorname{Re}\langle f, \xi \mu\rangle .
$$

Therefore

$$
\operatorname{Re}\langle f, \xi \mu\rangle=\int \operatorname{Re}\langle f, \xi s\rangle \mathrm{d} \mu(s)<\operatorname{Re}\langle f, \xi \mu\rangle,
$$

which is contradiction.

Let $\Omega$ be a subset of $M(S)$. We say that a net $\left(\mu_{\alpha}\right)$ in $M_{0}(S)$ is asymptotically $\Omega$ invariant if $\left\|\mu * \mu_{\alpha}-\mu_{\alpha}\right\| \rightarrow 0$ whenever $\mu \in \Omega$. Asymptotical invariance property was introduced by Day [4]. Wong [18] demonstrated that $M(S)^{*}$ has a topologically left invariant mean if and only if there exists a net $\left(\mu_{\alpha}\right)$ in $M_{0}(S)$ such that $\left\|\mu * \mu_{\alpha}-\mu_{\alpha}\right\| \rightarrow 0$ whenever $\mu \in M_{0}(S)$. If $S$ is a semi-foundation semigroup, the following conditions [7] are equivalent:

(a) $M(S)^{*}$ has a topologically left invariant mean; 
(b) there is a net $\left(\mu_{\alpha}\right)$ in $M_{0}(S)$ such that, for every compact subset $K$ of $S, \| \mu * \mu_{\alpha}-$ $\mu_{\alpha} \| \rightarrow 0$ uniformly over all $\mu$ in $M_{0}(S)$ which are supported on $K$.

Let $M(S)^{*}$ have a topologically left invariant mean, and let $\left(\mu_{\alpha}\right)$ be a net in $M_{0}(S)$. It is easy to see that $\left(\mu_{\alpha}\right)$ is asymptotically $M_{0}(S)$-invariant if and only if

$$
\lim _{\alpha}\left\|\mu * \mu_{\alpha}\right\|=|\mu(S)|
$$

whenever $\mu \in M(S)$.

Let $X$ be a right Banach $S$-module. Let $\left(\mu_{\alpha}\right)$ be an asymptotically $M_{0}(S)$-invariant in $M_{0}(S)$. For each $\alpha$, we define $E_{\alpha}[\xi] \in X$ by

$$
E_{\alpha}[\xi]=\int \xi s \mathrm{~d} \mu_{\alpha}(s), \quad \xi \in X
$$

We now concentrate on the mean ergodic theorem for a locally compact semigroup. The following result can be referred to as 'the mean ergodic theorem' for amenable locally compact semigroups (see [10[14]15], for details).

Theorem 1. Let $M(S)^{*}$ have a topologically right invariant mean. Suppose $\left(\mu_{\alpha}\right)$ is an asymptotically $M_{0}(S)$-invariant in $M_{0}(S)$.

(1) If $\xi \in X$ is such that $C_{\xi}=\overline{c o}\left\{\xi \delta_{s} ; s \in S\right\}$ is weakly compact, then $\left(E_{\alpha}[\xi]\right)$ converges to a fixed point of $C_{\xi}$ that is the unique fixed point in $C_{\xi}$, and is therefore independent of the choice of $\left(\mu_{\alpha}\right)$.

(2) Suppose that $C_{\xi}$ is weakly compact for each $\xi \in X$. Let $Y=\{\xi \in X$; $\xi s=\xi$ for all $s \in$ $S\}$, and let $Z$ be the closed vector subspace of $X$ generated by $\{\xi-\xi s ; s \in S, \xi \in X\}$. Then $X=Y \oplus Z$ and $\left(E_{\alpha}\right)$ converges strongly to the projection of $X$ onto $Y$.

(3) Let $C_{\xi}$ be weakly compact for any $\xi \in X$. If $P: X \rightarrow Y$ is the projection onto $Y$ associated with the direct sum decomposition of $(2)$, then $P(\xi)=Y \cap C_{\xi}$ for all $\xi \in X$.

Proof.

(1) Let $M$ be a topologically right invariant mean on $M(S)^{*}$. If $\mathscr{M}$ is the convex set of all means on $M(S)^{*}$, it is well known that $\mathscr{M}$ is weak* compact and that $M_{0}(S)$ is weak ${ }^{*}$ dense in $\mathscr{M}$ (see [311] for details). Therefore there is a net $\left(\mu_{\alpha}\right)$ in $M_{0}(S)$ such that $\mu_{\alpha} \rightarrow M$ in the weak* topology. A subnet $\left(\xi \mu_{\beta}\right)$ of $\left(\xi \mu_{\alpha}\right)$ converges in the weak topology to an element $\zeta$ in the compact subset $C_{\xi}=\overline{\left\{\xi \mu ; \mu \in M_{0}(S)\right\}}$. For every $s \in S$ and $f \in X^{*}$, we have

$$
\begin{aligned}
\langle f, \zeta s\rangle & =\langle s f, \zeta\rangle=\lim _{\beta}\left\langle s f, \xi \mu_{\beta}\right\rangle=\lim _{\beta}\left\langle\mu_{\beta}(s f), \xi\right\rangle=\lim _{\beta}\left\langle\left(\mu_{\beta} * \delta_{s}\right) f, \xi\right\rangle \\
& =\lim _{\beta}\left\langle\mu_{\beta}\left(\delta_{s} f\right), \xi\right\rangle=\lim _{\beta}\left\langle\mu_{\beta},\left(\delta_{s} f\right) \xi\right\rangle=\lim _{\beta}\left\langle\mu_{\beta}, \delta_{s}(f \xi)\right\rangle \\
& =\left\langle M, \delta_{s}(f \xi)\right\rangle=\langle M, f \xi\rangle=\lim _{\beta}\left\langle\mu_{\beta}, f \xi\right\rangle \\
& =\lim _{\beta}\left\langle\mu_{\beta} f, \xi\right\rangle=\lim _{\beta}\left\langle f, \xi \mu_{\beta}\right\rangle=\langle f, \zeta\rangle .
\end{aligned}
$$


So $\zeta s=\zeta$ for every $s \in S$, that is, $\zeta$ is a fixed point under the action of $S$. If $\varepsilon>0$, we determine $\alpha_{1}, \ldots, \alpha_{m}$ and $s_{1}, \ldots, s_{m} \in S$ such that $\sum_{i=1}^{m} \alpha_{i}=1$ and $\left\|\xi^{\prime}-\zeta\right\|<\varepsilon$, where $\xi^{\prime}=\sum_{i=1}^{m} \alpha_{i} \xi_{s_{i}}$. We have

$$
\begin{aligned}
\left\|E_{\alpha}[\xi]-\zeta\right\| & =\left\|\xi \mu_{\alpha}-\zeta\right\| \leq\left\|\xi \mu_{\alpha}-\xi^{\prime} \mu_{\alpha}\right\|+\left\|\xi^{\prime} \mu_{\alpha}-\zeta\right\| \\
& =\left\|\xi \sum_{i=1}^{m}\left(\alpha_{i} \mu_{\alpha}-\alpha_{i} \delta_{s_{i}} * \mu_{\alpha}\right)\right\|+\left\|\xi^{\prime} \mu_{\alpha}-\zeta \mu_{\alpha}\right\| \\
& \leq k\|\xi\| \sum_{i=1}^{m} \alpha_{i}\left\|\mu_{\alpha}-\delta_{s_{i}} * \mu_{\alpha}\right\|+k\left\|\xi^{\prime}-\zeta\right\| \\
& <k\|\xi\| \sum_{i=1}^{m}\left\|\mu_{\alpha}-\delta_{s_{i}} * \mu_{\alpha}\right\|+k \varepsilon
\end{aligned}
$$

Hence

$$
\lim _{\alpha}\left\|E_{\alpha}[\xi]-\zeta\right\|=0 .
$$

For every $\mu \in M_{0}(S)$, we have $E_{\alpha}[\xi \mu]=\xi \mu * \mu_{\alpha}$. Hence

$$
\begin{aligned}
\lim _{\alpha}\left\|E_{\alpha}[\xi \mu]-\zeta\right\| & =\lim _{\alpha}\left\|\xi \mu * \mu_{\alpha}-\xi \mu_{\alpha}\right\| \\
& \leq k \lim _{\alpha}\|\xi\|\left\|\mu * \mu_{\alpha}-\mu_{\alpha}\right\|=0
\end{aligned}
$$

and $\lim _{\alpha}\left\|E_{\alpha}[\varsigma]-\zeta\right\|=0$ for every $\varsigma \in C_{\xi}$. Now if there exists another fixed point $\zeta^{\prime}$ in $C_{\xi}$, then for every $\alpha$ we would have

$$
E_{\alpha}\left[\zeta^{\prime}\right]=\int \zeta^{\prime} s \mathrm{~d} \mu_{\alpha}(s)=\zeta^{\prime}
$$

and

$$
\zeta^{\prime}=\lim _{\alpha} E_{\alpha}\left[\zeta^{\prime}\right]=\zeta .
$$

This shows that $\left(E_{\alpha}[\xi]\right)$ converges to the unique fixed point $\zeta$ in $C_{\xi}$. Now if there exists another asymptotically $M_{0}(S)$-invariant $\left(v_{\beta}\right)$ in $M_{0}(S)$, it is easy to see that $\lim _{\beta} E_{\beta}[\xi]=$ $\zeta$.

(2) Define an operator $P: X \rightarrow X$ by $P(\xi)=\lim _{\alpha} E_{\alpha}[\xi]$. Since $X$ is a right $S$-module, it is easy to see that $\|P\| \leq k$. For every $\xi \in X, P(\xi) \in Y$ and $P(P(\xi))=\lim _{\alpha} P(\xi) \mu_{\alpha}=P(\xi)$, $P^{2}=P$. If $\xi \in Y$, then $P(\xi)=\lim _{\alpha} E_{\alpha}[\xi]=\xi \in Y$. We conclude that $Y$ is the closed subspace $P(X)$ of $X$. Let $\xi \in X$ and $s \in S$. We have

$$
\begin{aligned}
\|P(\xi-\xi s)\| & =\left\|\lim _{\alpha} E_{\alpha}[\xi-\xi s]\right\|=\lim _{\alpha}\left\|\xi \mu_{\alpha}-\xi \delta_{s} * \mu_{\alpha}\right\| \\
& \leq k\|\xi\| \lim _{\alpha}\left\|\delta_{s} * \mu_{\alpha}-\mu_{\alpha}\right\|=0 .
\end{aligned}
$$

So $\xi-\xi \delta_{s} \in Y^{\perp}$. Conversely if $\zeta \in Y^{\perp}, P(\zeta)=0$ is the unique fixed point in $C_{\zeta}$ and, for every $\varepsilon>0$, there exist $\alpha_{1}, \ldots, \alpha_{m}$ and $s_{1}, \ldots, s_{m}$ in $S$ such that $\sum_{i=1}^{m} \alpha_{i}=1$ and $\left\|\sum_{i=1}^{m} \alpha_{i} \zeta \delta_{s_{i}}\right\|<\varepsilon$. Hence $\left\|\zeta-\sum_{i=1}^{m} \alpha_{i}\left(\zeta-\zeta \delta_{s_{i}}\right)\right\|=\left\|\sum_{i=1}^{m} \alpha_{i} \zeta \delta_{s_{i}}\right\|<\varepsilon$, such that $\zeta \in Z$. (3) This is trivial. 


\section{COROLLARY 1.}

Let $M(S)^{*}$ have a topologically right invariant mean. Suppose further that $\left(\mu_{\alpha}\right)$ is an asymptotically $M_{0}(S)$-invariant in $M_{0}(S)$. Suppose that $S$ acts from the right on a reflexive Banach space $X$. Then $\left(E_{\alpha}[\xi]\right)$ converges to an element $\zeta$ in $X$ that is $S$-invariant.

Proof. For every $\xi \in X$, the set $C_{\xi}=\overline{c o}\{\xi s ; s \in S\}$ is a weak ${ }^{*}$-closed subset of the closed ball of radius $k\|\xi\|$ in $\left(X^{*}\right)^{*}$. This ball is weak* compact by Alaoglu's theorem and hence $C_{\xi}$ is weak compact in the reflexive space $X$ (p. 111 of [17]). The statement is an immediate consequence of Theorem 1 .

We recall that, for a semigroup $S, M_{a}(S)$ is the set of all measures $\mu \in M(S)$ such that both mappings $s \mapsto|\mu| * \delta_{s}$ and $s \mapsto \delta_{s} *|\mu|$ from $S$ into $M(S)$ are weakly continuous (see [1] and [5] for definition). A semigroup $S$ is called a foundation semigroup if $\bigcup\left\{\operatorname{supp} \mu ; \mu \in M_{a}(S)\right\}$ is dense in $S$. It is known that $M_{a}(S)$ admits a positive approximate identity with norm 1 [5].

A functional $f$ in $M_{a}(S)^{*}$ is said to be weakly almost periodic if the set $\{f \mu ; \mu \in$ $\left.M_{a}(S),\|\mu\| \leq 1\right\}$ is relatively weakly compact. We denote by $\operatorname{wap}\left(M_{a}(S)\right)$ the closed subspace of $M_{a}(S)^{*}$ consisting of all the weakly almost periodic functionals in $M_{a}(S)^{*}$ (for more on weakly almost periodic functionals, the reader is referred to [2] and [12]).

\section{COROLLARY 2.}

Let $S$ be a foundation, locally compact Hausdorff topological semigroup with identity. Let $M(S)^{*}$ have a topologically right invariant mean. Suppose $\left(\mu_{\alpha}\right)$ is an asymptotically $M_{0}(S)$-invariant in $M_{0}(S)$.

(1) If $f \in \operatorname{wap}\left(M_{a}(S)\right)$, then $E_{\alpha}[f]$ converges to an element $f^{\prime}$ in $\operatorname{wap}\left(M_{a}(S)\right)$ that is $S$ invariant, that is, $f^{\prime} s=s$ for all $s \in S ; f^{\prime}$ is independent of the choice of $\left(\mu_{\alpha}\right)$.

(2) Let $M$ be the topologically left invariant mean on wap $\left(M_{a}(S)\right)$. Then $Y=\mathbb{C} 1$ and

$$
Z=\left\{f \in \operatorname{wap}\left(M_{a}(S)\right) ;\langle M, f\rangle=0\right\}
$$

Note that if $\left(\mu_{\alpha}\right)$ is an asymptotically $M_{0}(S)$-invariant in $M_{0}(S)$, then

$$
\left\|\mu * \mu_{\alpha}-\mu_{\alpha}\right\| \rightarrow 0
$$

for all $\mu \in M_{0}(S)$. We choose $v \in M_{a}(S) \cap M_{0}(S)$ and let $v_{\alpha}=\mu_{\alpha} * v, \alpha \in I$. Clearly $\left\|\mu * v_{\alpha}-v_{\alpha}\right\| \rightarrow 0$ for all $\mu \in M_{0}(S)$. If $M$ is a weak* cluster point of $\left(v_{\alpha}\right)$, then $M$ is a topologically left invariant mean on $\operatorname{wap}\left(M_{a}(S)\right)$.

Proof.

(1) We apply Theorem 1 to the Banach space $X=\operatorname{wap}\left(M_{a}(S)\right)$. If $f \in \operatorname{wap}\left(M_{a}(S)\right)$ and $s \in S$, let

$$
\langle f s, \mu\rangle=\left\langle f, \delta_{s} * \mu\right\rangle, \quad \mu \in M_{a}(S) .
$$

We check the continuity of the mapping $s \rightarrow f s$ for any $f \in \operatorname{wap}\left(M_{a}(S)\right)$. Let $f \in \operatorname{wap}\left(M_{a}(S)\right)$ and $\left(e_{\alpha}\right)$ be a bounded approximate identity in $M_{a}(S)$. It is easy to see that $f e_{\alpha} \rightarrow f$ in the weak* topology. By the compactness of $\left\{f \mu ; \mu \in M_{a}(S),\|\mu\| \leq 1\right\}$, 
we can suppose that $f e_{\alpha} \rightarrow f$ in the weak topology, passing to a subnet, if necessary. Clearly $f \in M_{a}(S)^{*} M_{a}(S)$, since $M_{a}(S)^{*} M_{a}(S)$ is a Banach space and $f e_{\alpha} \in M_{a}(S)^{*} M_{a}(S)$ for all $\alpha \in I$. By Lemma 2.2 in [6], $s \mapsto f s$ is continuous. Obviously $X$ is a right Banach $S$-module.

If $f \in \operatorname{wap}\left(M_{a}(S)\right)$, then $\{f s ; s \in S\}$ is relatively weakly compact. So Theorem 1 asserts that, for every $f \in X,\left(E_{\alpha}[f]\right)$ converges to an element $f^{\prime}$ in $X$ that is fixed; $f^{\prime} s=f^{\prime}$ and $\left\langle f^{\prime}, \delta_{s} * \mu\right\rangle=\left\langle f^{\prime}, \mu\right\rangle$ for every $s \in S$ and every $\mu \in M_{a}(S)$.

(2) By Theorem 1, $Y$ is the set of all $f \in X$ such that $f s=f$ for all $s \in S$. If $f \in Y$, then $\left\langle f, \delta_{s} * \mu\right\rangle=\langle f, \mu\rangle$ for all $s \in S$ and $\mu \in M_{a}(S)$. Consequently, $f=\left\langle f, \delta_{e}\right\rangle 1$.

We know that $Z$ admits a dense subset consisting of functionals of the form $g=\Sigma_{1}^{n} f_{i}-$ $f_{i} s_{i}$, where $f_{i} \in \operatorname{wap}\left(M_{a}(S)\right)$ and $s_{i} \in S$. By the definition of $M$, we have $\langle M, g\rangle=0$ and so $Z \subseteq\left\{f \in \operatorname{wap}\left(M_{a}(S)\right) ;\langle M, f\rangle=0\right\}$. For the reverse inclusion, let $f \in \operatorname{wap}\left(M_{a}(S)\right)$ and $\langle M, f\rangle=0$. By Theorem 1, we can write $f=f_{1}+f_{2}$ for some $f_{1} \in Y$ and $f_{2} \in Z$, and so

$$
\begin{aligned}
0 & =\langle M, f\rangle=\left\langle M, f_{1}\right\rangle+\left\langle M, f_{2}\right\rangle \\
& =\left\langle M, f_{1}\right\rangle+0=\left\langle M, f_{1}\right\rangle,
\end{aligned}
$$

since $Z \subseteq\left\{f \in \operatorname{wap}\left(M_{a}(S)\right) ;\langle M, f\rangle=0\right\}$. Consequently $\left\langle M, f_{1}\right\rangle=0$. For each $\mu \in M_{0}(S)$, we have

$$
\begin{aligned}
0 & =\left\langle M, f_{1}\right\rangle=\left\langle M, f_{1} \mu\right\rangle=\lim _{\alpha}\left\langle\mu_{\alpha}, f_{1} \mu\right\rangle \\
& =\lim _{\alpha}\left\langle f_{1}, \mu * \mu_{\alpha}\right\rangle=\lim _{\alpha}\left\langle f_{1} \mu * \mu_{\alpha}, \delta_{e}\right\rangle \\
& =\lim _{\alpha}\left\langle f_{1} \mu, \delta_{e}\right\rangle=\left\langle f_{1}, \mu\right\rangle .
\end{aligned}
$$

Therefore $f_{1}=0$. It follows that $Z=\left\{f \in \operatorname{wap}\left(M_{a}(S)\right) ;\langle M, f\rangle=0\right\}$.

\section{Acknowledgment}

I would like to thank the referee for his/her careful reading of my paper and many valuable suggestions.

\section{References}

[1] Amini M and Medghalchi A R, Fourier algebras on topological foundation *-semigroup, Semigroup Forum 68 (2004) 322-334

[2] Baker J, Lau A T and Pym J S, Module homomorphisms and topological centres associated with weakly sequentially complete Banach algebras, J. Funct. Anal. 158 (1998) 186-208

[3] Berglund J F, Junghenn H D and Milnes P, Analysis on semigroups, function spaces, compactifications, representions (New York) (1989)

[4] Day M M, Means on semigroups and groups, Bull. Amer. Math. Soc. 55 (1949) 10541055

[5] Dzinotyiweyi H A M, The analogue of the group algebra for topological semigroups, (London: Pitman, Boston) (1984)

[6] Ghaffari A, Convolution operators on semigroup algebras, Southeast Asian Bull. Math. 27 (2003) 1-12 
[7] Ghaffari A, Topologically left invariant mean on dual semigroup algebras, Bull. Iranian Math. Soc. 28 (2002) 69-75

[8] Ghaffari A, Topologically left invariant mean on semigroup algebras, Proc. Indian Acad. Sci. (Math. Sci.) 115 (2005) 453-459

[9] Greenleaf F P, Ergodic theorems and the construction of summing sequences in amenable locally compact groups, Comm. Pure Apple. Math. 26 (1973) 29-46

[10] Greenleaf F P, Invariant means on topological groups (New York: Van Nosstrand) (1969)

[11] Lau A T, Amenability of semigroups, The analytical and topological theory of semigroups (eds) K H Hofman, J D Lawson and J S Pym (Berlin and New York: Walter de Gruyter) (1990) pp. 331-334

[12] Lau A T and Ulger A, Topological centers of certain dual algebras, Trans. Am. Math. Soc. 348 (1996) 1191-1212

[13] Lo Bello A, On the origin and history of ergodic theory Boll. Storia Sci. Mat. 3 (1983) 37-75

[14] Paterson A L T, Amenability, Amer. Math. Soc. Math. Survey and Monographs 29 (Rhode Island: Providence) (1988)

[15] Pier J P, Amenable locally compact groups (New York: John Wiley And Sons) (1984)

[16] Riazi A and Wong J C S, Characterizations of amenable locally compact semigroups, Pacific J. Math. 108 (1983) 479-496

[17] Rudin W, Functional analysis (New York: McGraw Hill) (1991)

[18] Wong J C S, An ergodic property of locally compact semigroups, Pacific J. Math. 48 (1973) 615-619

[19] Wong J C S, A characterization of topological left thick subsets in locally compct left amenable semigroups, Pacific J. Math. 62 (1976) 295-303

[20] Wong J C S, Fixed point theorems for measurable semigroups of operations, Canad. J. Math. 44 (1992) 652-664 